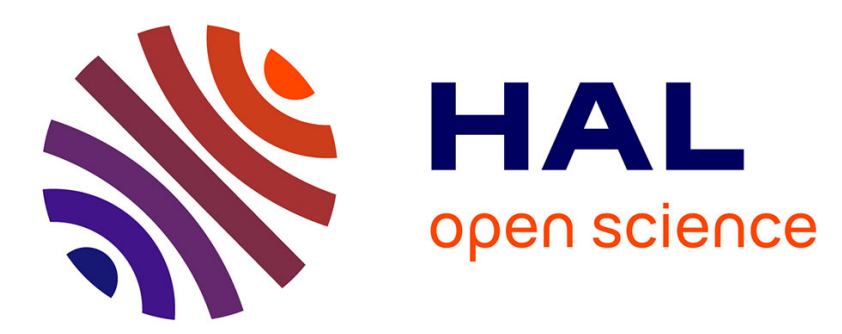

\title{
Effets immunotoxiques des polluants environnementaux : exemple des hydrocarbures aromatiques polycycliques \\ Lydie Sparfel
}

\section{- To cite this version:}

Lydie Sparfel. Effets immunotoxiques des polluants environnementaux : exemple des hydrocarbures aromatiques polycycliques. Archives des Maladies Professionnelles et de L'Environnement, 2016, 77 (6), pp.990-997. 10.1016/j.admp.2016.09.003 . hal-01467562

HAL Id: hal-01467562 https://hal-univ-rennes1.archives-ouvertes.fr/hal-01467562

Submitted on 23 May 2017

HAL is a multi-disciplinary open access archive for the deposit and dissemination of scientific research documents, whether they are published or not. The documents may come from teaching and research institutions in France or abroad, or from public or private research centers.
L'archive ouverte pluridisciplinaire $\mathbf{H A L}$, est destinée au dépôt et à la diffusion de documents scientifiques de niveau recherche, publiés ou non, émanant des établissements d'enseignement et de recherche français ou étrangers, des laboratoires publics ou privés. 
Effets immunotoxiques des polluants environnementaux : exemple des hydrocarbures aromatiques polycycliques

Immunotoxic effects of environmental pollutants such as polycyclic aromatic hydrocarbons

\section{Sparfel}

UMR INSERM U1085, Institut de recherche en santé, environnement et travail, Université de Rennes 1 (IRSET), 2 avenue du Pr Léon Bernard, 35043 Rennes, France e-mail : lydie.sparfel@univ-rennes1.fr 
Immunotoxic effects of environmental pollutants such as polycyclic aromatic hydrocarbons

\section{Summary}

The immune system contributes to the monitoring and maintains the integrity of the organism. It can be the target of many xenobiotics, and immunotoxicology attempts to analyze the immunological alterations caused by exposure to these xenobiotics. Several environmental pollutants are strongly suspected of acting on the immune system to cause a decrease in the organism protective functions. Thus, polycyclic aromatic hydrocarbons, well known for their carcinogenic effects, have also been shown to induce immunosuppressive effects, proinflammatory actions and some hypersensitivity reactions, involved in the development of various pathologies. These different immunotoxic effects were mainly identified by experimental studies in animals and the transposition of these effects to humans remains incomplete. To better understand the immunotoxic effects of exposure to environmental pollutants and their effects on human health, it is appropriate to strengthen immunotoxicology studies in human.

Key words : immune system, immunotoxicology, environmental pollutant, polycyclic aromatic hydrocarbons 
Effets immunotoxiques des polluants environnementaux : exemple des hydrocarbures aromatiques polycycliques

\section{Résumé}

Le système immunitaire contribue à la surveillance et au maintien de l'intégrité de l'organisme. Il peut être la cible de nombreux xénobiotiques, et l'immunotoxicologie s'attache à étudier les différentes atteintes immunologiques induites par l'exposition à ces xénobiotiques. De nombreux polluants environnementaux sont fortement suspectés d'agir sur le système immunitaire pour entraîner une diminution des fonctions de protection de l'organisme. Ainsi, les hydrocarbures aromatiques polycycliques, bien connus pour leurs effets cancérogènes, se sont révélés également capables d'induire des effets immunosuppresseurs, d'entraîner des actions pro-inflammatoires et de majorer certaines réactions d'hypersensibilité, impliqués dans le développement de diverses pathologies. Ces différents effets immunotoxiques ont surtout été identifiés par des études expérimentales chez l'animal et la transposition de ces effets à l'homme reste incomplète. Pour mieux appréhender les effets immunotoxiques de l'exposition aux polluants environnementaux et leurs conséquences sur la santé humaine, il convient donc de renforcer les études en immunotoxicologie chez l'homme.

Mots clés : système immunitaire, immunotoxicologie, polluants environnementaux, hydrocarbures aromatiques polycycliques 


\section{Introduction}

Le système immunitaire joue un rôle clé dans la surveillance et le maintien de l'intégrité de l'organisme en éliminant, neutralisant ou permettant la co-existence avec toute substance étrangère à celui-ci (virus, bactéries, parasites, champignons, cellules tumorales ...). Les constituants du système immunitaire sont disséminés dans tout l'organisme et sont capables d'interagir avec les autres grands systèmes physiologiques expliquant ainsi que la perturbation du système immunitaire peut être à l'origine de graves désordres pathologiques.

\section{Le système immunitaire}

Le système immunitaire est formé d'un ensemble complexe d'organes et de tissus comprenant classiquement les organes centraux ou primaires (moelle osseuse et thymus ayant un rôle clé dans la maturation et l'éducation des lymphocytes), les organes périphériques ou secondaires (ganglions lymphatiques, rate et organes lymphoïdes associés aux muqueuses qui concentrent les antigènes et permettent la coopération cellulaire en vue de l'orientation de la réponse immunitaire) et les systèmes sanguin et lymphatique. Le système immunitaire est un système intégré mettant en jeu des cellules immunocompétentes produites dans la moelle osseuse et issues d'un précurseur commun, la cellule souche hématopoïétique exprimant le marqueur CD34, douée de propriétés d'autorenouvèlement et de différentiation en progéniteurs lymphoïde et myéloïde, à l'origine de précurseurs, premières cellules morphologiquement orientées pour chaque lignée, donnant ensuite les cellules matures possédant des lieux de résidence bien spécifiques. Ainsi, circulent dans notre sang, les cellules issues du progéniteur lymphoïde : les lymphocytes B (maturation dans la moelle), T (maturation dans le thymus) et NK (Natural Killer), et celles issues du progéniteur myéloïde, les granulocytes (encore appelés polynucléaires), monocytes, plaquettes et globules rouges, 
tandis que les macrophages, les cellules dendritiques, les mastocytes et les plasmocytes résident dans nos tissus.

Les éléments de défense du système immunitaire interviennent dans 3 types d'immunité : l'anté-immunité, l'immunité innée et l'immunité spécifique. L'anté-immunité représente la première ligne de défense de l'organisme et constitue une réponse immédiate pour éviter que le système immunitaire ne soit mis à contribution. Elle est basée sur les barrières physiques, chimiques ou microbiologiques formées par la peau, les muqueuses des voies respiratoires et uro-génitale, ou encore l'acidité des voies gastro-intestinales. Cette antéimmunité ne possède pas de mémoire immunologique. La seconde ligne de défense est représentée par l'immunité innée encore appelée immunité naturelle ou non spécifique (Figure 1). Celle-ci peut être mobilisée lors de la mise en contact de substances étrangères avec les fluides corporels (sang, lymphe, liquide interstitiel...) en cas d'agression (blessure, opération, forte dose de xénobiotiques...) et est associée à une inflammation. Les composants cellulaires de l'immunité innée sont les cellules immunes capables de phagocytose comme les granulocytes neutrophiles et les cellules mononucléées de la lignée monocyte/macrophage ainsi que les cellules présentatrices d'antigène telles que les cellules dendritiques et les macrophages ou encore les lymphocytes NK, avec pour fonction de se débarrasser des cellules infectées ou tumorales. A côté de cette réponse cellulaire, l'immunité innée peut aussi mettre en jeu une réponse humorale basée sur l'activation du complément ou l'intervention de cytokines. Les composants cellulaires et humoraux de l'immunité innée sont dotés d'une existence préalable et sont rapidement mobilisés après agression. La troisième ligne de défense est constituée par l’immunité adaptative, dite immunité spécifique ou acquise à un antigène particulier (Figure 1). Elle est basée sur une reconnaissance spécifique des antigènes par les récepteurs des lymphocytes B et $\mathrm{T}$, acteurs majeurs de cette immunité spécifique et dépend de l'activation, de la multiplication et de la différenciation de ces lymphocytes. Les 
lymphocytes B peuvent être activés directement par l'antigène et sont responsables de la réponse humorale caractérisée par la production d'anticorps par les plasmocytes ou par la formation de lymphocytes $\mathrm{B}$ mémoires. Les lymphocytes $\mathrm{T}$ ont besoin d'une présentation antigénique par les cellules dendritiques, les lymphocytes B ou les macrophages pour induire une réponse cellulaire. Cette réponse cellulaire se caractérise par la survenue d'une cytotoxicité menée par les lymphocytes cytotoxiques (Tc) encore appelés CD8+ ou d'une régulation orchestrée par les lymphocytes T auxiliaires ou helper (Th) encore appelés CD4+, capables de se différencier selon le contexte environnemental en sous-populations distinctes les plus adaptées à l'agression, on y trouve les Th1, les Th2, les Th17 et les T régulateurs (Treg). Les lymphocytes Th1 favorisent une réponse immunitaire cellulaire anti-infectieuse en coopérant et activant les différentes cellules cytotoxiques (lymphocytes Tc, NK, macrophages). Les lymphocytes Th2 orientent la réponse vers l'immunité à médiation humorale faisant intervenir la lignée B et la production d'anticorps de haute affinité ; cette réponse Th2 est souvent associée à des manifestations d'allergies. Les lymphocytes Th17, découverts plus récemment, sont caractérisés par leur capacité à produire une cytokine fortement pro-inflammatoire, l'interleukine (IL)-17. A l'inverse, les Treg sont des cellules immunosuppressives qui vont contrôler et limiter la réponse immunitaire. Dans de nombreux contextes ainsi que dans diverses pathologies, les réponses immunes induites associent des réponses innées et spécifiques ; c'est le cas par exemple dans l'asthme où la perturbation de la barrière épithéliale par une infection ou un polluant, constitue le premier signal de danger à l'origine de l'activation de l'immunité innée, par la prise en charge de l'antigène par les cellules dendritiques présentes sous la membrane basale de l'épithélium bronchique, conduisant à la maturation de ces cellules dendritiques et à leur migration dans les ganglions médiastinaux qui drainent les poumons où elles présenteront l'antigène aux lymphocytes $\mathrm{T}$ dits naïfs pour initier leur conversion en lymphocytes plutôt Th2 et donc la réponse immune 
adaptative [1]. Par ailleurs, de nombreuses molécules peuvent porter atteinte au système immunitaire et altérer l'immunité innée et l'immunité adaptative entraînant une diminution de la capacité de surveillance de l'organisme. Les conséquences de telles altérations sont une augmentation de l'incidence des infections, la survenue d'allergies et une diminution de la résistance aux tumeurs malignes.

\section{L’immunotoxicologie}

L'immunotoxicologie se définit comme l'étude des effets délétères induits par l'exposition à un xénobiotique (médicaments, pesticides, produits industriels, polluants, additifs alimentaires, cosmétiques ...) sur le système immunitaire. Elle s'intéresse à mettre en évidence les perturbations immunologiques chez l'animal ou l'homme, à comprendre les mécanismes en cause dans ces perturbations et à évaluer les risques pour les personnes concernées. Si l'immunotoxicologie bénéficie aujourd'hui de connaissances relativement approfondies des effets chez l'animal, les phénomènes immunotoxiques chez l'homme restent plus méconnus et moins bien décrits. De plus, les modèles expérimentaux utilisés en immunotoxicologie ne permettent pas, à ce jour, de valider complètement l'extrapolation des résultats obtenus chez l'animal à l'homme. En immunotoxicologie, on distingue classiquement 4 grands types de réactions : l'immunosuppression, l'immunostimulation, les réactions d'hypersensibilité et celles d'auto-immunité L'immunosuppression est définie comme une inhibition de la réponse immunitaire. Elle a été documentée par des études expérimentales chez l'animal, comme par exemple celles réalisées chez les rongeurs exposés à la 2,3,7,8-tétrachlorodibenzo-para-dioxine, encore appelée dioxine de Seveso depuis l'accident de l'usine de pesticides survenu en Italie en 1976, rapportant une involution thymique et une inhibition plus ou moins importante de l'immunité humorale avec toutefois une incidence limitée sur la susceptibilité aux infections [2]. Chez l'homme, 
l'immunosuppression a surtout été documentée à partir des études réalisées chez les transplantés traités par des médicaments immunosuppresseurs ou encore chez les personnes atteintes de maladies auto-immunes. Les conséquences d'une immunosuppression sont prévisibles et bien connues, il s'agit d'une susceptibilité accrue aux infections, aux tumeurs d'origine virale (lymphomes malins non hodgkiniens, sarcomes de Kaposi, cancers du col de l'utérus...). L'immunostimulation, encore appelée immunoactivation, est la stimulation du système immunitaire aboutissant à une manifestation pathologique. Elle est liée à l'introduction récente des médicaments immunostimulants et les manifestations, moins bien documentées que celles de l'immunosuppression, sont des réactions hyperthermiques (syndromes pseudo-grippaux, syndrome aigu des cytokines), ou l'aggravation de pathologies immunitaires préexistantes sous forme de réactions allergiques ou de maladies autoimmunes). Le syndrome aigu des cytokines est classiquement associé à l'administration de cytokines recombinantes (interféron- $\gamma$, IL-2) ou d'anticorps monoclonaux, comme par exemple, l'administration d'un anticorps monoclonal anti-CD28, contribuant à l'activation des lymphocytes T, ayant conduit au cours d'un essai clinique en 2006, au développement d'un syndrome inflammatoire généralisé, rapidement suivi par une atteinte pulmonaire et rénale et une coagulopathie intravasculaire disséminée [3]. A ce jour, la prédictivité des modèles non cliniques reste plutôt limitée pour évaluer une immunostimulation. $\underline{\text { Les }}$ réactions d'hypersensibilité se caractérisent comme des réactions immunitaires intenses et inappropriées de l'organisme à une substance étrangère à celui-ci. On en distingue 4 classes : les réactions d'hypersensibilité immédiate caractérisées par des anticorps (Immunoglobulines (Ig) de classe E (IgE)) se fixant sur les mastocytes tissulaires entraînant des symptômes d'apparition rapide ; les réactions d'hypersensibilité cytotoxique où les anticorps (IgG, $\operatorname{IgM})$ reconnaissent les antigènes à la surface des cellules et activent le complément produisant une cytolyse ; les réactions d'hypersensibilité semi-retardée dépendantes des complexes immuns 
induisant des lésions tissulaires après activation du complément et les réactions d'hypersensibilité retardée médiées par les lymphocytes $\mathrm{T}$ et les cytokines induisant des lésions tissulaires inflammatoires. Les conséquences cliniques de ces réactions peuvent alors être l'anaphylaxie ou encore diverses manifestations cutanées (toxidermies, urticaires, eczémas de contact...), hématologiques (anémies hémolytiques, neutropénies, agranulocytose...), respiratoires (asthme...), hépatiques (hépatites immuno-allergiques...) ou rénales (glomérulonéphrites, néphrites immuno-allergiques...). Les réactions d'autoimmunité se définissent comme des réponses immunitaires dirigées contre les composants normaux de l'organisme. Les mécanismes impliqués sont une rupture de la tolérance au soi ou une modification d'auto-antigènes par fixation covalente d'un métabolite réactif, conduisant à l'activation des lymphocytes $\mathrm{B}$ et $\mathrm{T}$ produisant des anticorps ou des cellules effectrices reconnaissant les constituants du soi et responsables de lésions cellulaires et tissulaires. Les conséquences cliniques sont des réactions généralisées comme par exemple dans le cas d'un lupus érythémateux disséminé ou des réactions plus spécifiques d'organes telles que celles observées dans les anémies hémolytiques, les thrombopénies ou encore les hépatites.

\section{Les effets immunotoxiques des HAPs}

\section{Les HAPs}

Ces dernières années, l'exposition humaine à de nombreux polluants environnementaux est devenue une préoccupation majeure en santé publique. Parmi les contaminants environnementaux, les hydrocarbures aromatiques polycycliques (HAPs) constituent une famille de composés organiques très répandus. Ils représentent une grande famille regroupant à ce jour un nombre d'HAPs identifiés largement supérieur à 100 et présentent la particularité de se trouver dans l'environnement sous forme de mélanges plus ou moins complexes. Certains HAPs présentant des effets cancérogènes pour l'homme, cette 
famille de composés chimiques fait l'objet d'une attention particulière. Les HAPs sont constitués d'au moins 2 cycles aromatiques juxtaposés formés de carbone et d'hydrogène et ont pour chef de file le benzo $(a)$ pyrène $(\mathrm{B} a \mathrm{P})$. Ils sont principalement générés pendant la pyrolyse ou la combustion incomplète des matières organiques : ils peuvent avoir une origine naturelle (feux de forêts, de prairies ou éruptions volcaniques), mais ont surtout une origine anthropique impliquant des processus industriels tels que les combustions de pétrole et de charbon, les incinérations des déchets ou encore des processus urbains comme les combustions domestiques ou les fonctionnements des moteurs à essence et diesels. Ils sont aussi présents dans les produits alimentaires grillés ou fumés et dans la fumée de tabac [4]. Du fait de cette répartition ubiquiste, les expositions humaines aux HAPs sont non seulement d'origine professionnelle (cokeries, fonderies, raffinage du pétrole, pose d'enrobés routiers, de bitumes, affinage de l'aluminium), mais également d'origine domestique, notamment via l'alimentation qui représente près de $70 \%$ des expositions quotidiennes (traitement thermique des viandes et poissons, dépôt sur les fruits et légumes lors de pollution atmosphérique) ou via la pollution extérieure ou intérieure (tabagisme, combustion du bois, circulation automobile...). Le mécanisme général d'action moléculaire des HAPs a été relié à un récepteur cytosolique, le récepteur Ah (Aryl hydrocarbon) (RAh), auquel ils se lient, entraînant la translocation du complexe RAh-HAP dans le noyau et sa fixation sur des éléments de réponse aux xénobiotiques spécifiques de gènes cibles. Parmi ces gènes, se trouvent les cytochromes $\mathrm{P} 450$ 1A1, 1A2 et 1B1, nécessaires à la bioactivation de certains HAPs, comme le $\mathrm{B} a \mathrm{P}$, en métabolites oxydés électrophiles, qui peuvent alors se lier de façon covalente à des sites nucléophiles de l'ADN et ainsi former des adduits. Si ces lésions de l'ADN sont mal réparées, cela peut engendrer la formation d'une mutation, qui peut ensuite être transmise aux cellules filles lors de la mitose [5]. Ces dommages génotoxiques peuvent ainsi conduire à des effets cancérogènes bien documentés par des études expérimentales chez 
l'animal au niveau de différents tissus comme la peau, le poumon, le foie ou encore le système hématopoïétique [6]. Les résultats d'études épidémiologiques sur l'exposition professionnelle aux HAPs ont aussi mis en évidence une augmentation du risque de survenue de cancers du poumon chez les travailleurs exposés aux fumées de goudrons ou chez les ouvriers des cokeries, des cancers cutanés liés à la gazéification du charbon ou à la distillation de la houille et des cancers de la vessie chez les travailleurs des unités de production d'aluminium (tableaux 16bis et 36 bis des maladies professionnelles). En population générale, une corrélation fortement significative a également été décrite entre consommation de tabac et développement de cancers pulmonaires. A côté de leurs effets cancérogènes largement décrits, les HAPs se sont révélés capables d'exercer des effets immunotoxiques pour lesquels les connaissances restent plus restreintes et incomplètes, en particulier chez l'homme.

\section{Les effets immunosuppresseurs des HAPs}

De manière générale, les HAPs sont plutôt considérés comme des composés immunosuppresseurs. Ces effets ont surtout été documentés par des études expérimentales menées in vivo chez l'animal et in vitro chez l'animal et l'homme et ont beaucoup concerné l'immunité humorale. Connus depuis les années 1950 sous la forme de perturbation de l'immunité humorale via la diminution des niveaux d'hémolysine anti-hématies de mouton après exposition aigue à certains HAPs comme le 3-méthylcholanthrène, le benz( $a$ )anthracène ou le dibenz $(a, h)$ anthracène, l'immunosuppression a ensuite été mise en évidence par des travaux montrant que l'exposition subchronique à certains HAPs, en particulier le diméthylbenz( $a$ )anthracène (DMBA), était associée à une dépression de l'immunité humorale et de l'immunité cellulaire avec pour conséquence une susceptibilité accrue au développement tumoral et aux infections comme celles à Listeria monocytogenes chez la souris [7,8]. En effet dans ces études, le DMBA s'est révélé capable d'inhiber in vitro et in vivo la réponse des lymphocytes B activés par les lymphocytes T, ainsi que celle indépendante de ces derniers 
[7,8]. Par ailleurs, les effets inhibiteurs des HAPs sur l'immunité humorale ont été bien documentés : par exemple, le $\mathrm{B} a \mathrm{P}$ inhibe la production d'anticorps par les lymphocytes $\mathrm{B}$ in vivo chez la souris et in vitro dans des splénocytes de souris et dans des cellules sanguines de travailleurs exposés aux HAPs [9-11]. Une inhibition par le $\mathrm{B} a \mathrm{P}$ de la lymphopoïèse $\mathrm{B}$, ainsi qu'une apoptose par le DMBA des cellules précurseurs de ces lymphocytes B ont également été rapportées chez la souris, suggérant que le système immunitaire en développement serait une cible de la toxicité des HAPs [12,13]. Les effets des HAPs s'exercent aussi sur l'immunité cellulaire, mais les données les concernant sont plus restreintes. L'exposition à certains HAPs, comme le DMBA et le benzo $(k)$ fluoranthène a été associée à une perturbation de la réponse $\mathrm{T}$ cytotoxique et anti-tumorale, ainsi qu'à atrophie thymique liée à une déplétion en lymphocytes $\mathrm{T}$ chez la souris $[7,14]$. Ces effets ont notamment été reliés à l'inhibition de la sécrétion de l'IL-2, cytokine nécessaire à la prolifération des lymphocytes T [14]. Si les effets immunosuppresseurs des HAPs ont bien été décrits chez l'animal, leurs actions sur la fonction lymphocytaire humaine restent moins bien documentées. Chez l'homme, l'exposition aux HAPs se traduit par la présence de nombreux adduits à l'ADN dans les lymphocytes et le niveau de ces adduits a été associé à un risque de développement de certains cancers [15-17]. Les HAPs se sont enfin révélés capables d'altérer l'immunité innée. En effet, l'exposition au $\mathrm{B} a \mathrm{P}$ ou au 3-méthylcholanthrène a été associée à une inhibition de la présentation antigénique par les macrophages, à une inhibition de l'interaction de ces derniers avec les lymphocytes T et à une diminution de leur capacité de phagocytose chez la souris [18-20]. Chez l'homme, des études plus récentes menées in vitro ont mis en évidence une inhibition par le $\mathrm{B} a \mathrm{P}$ de la formation des cellules dendritiques et des macrophages à partir de monocytes humains isolés du sang périphérique ainsi qu'une apoptose des macrophages humains différenciés [21-23]. Dans les études sur les expositions professionnelles aux HAPs, de légères modifications de la fonction immunitaire telles que la diminution du taux d'IgG et d'IgA ont été observées sans 
qu'il y ait toutefois de signes cliniques de troubles immunitaires [11]. Les effets des HAPs sur l'immunité innée et sur l'immunité spécifique humorale et cellulaire apparaissent donc divers et variés (Figure 1), les mécanismes sous-jacents restent cependant incomplètement élucidés. Il a généralement été observé que les HAPs les plus cancérogènes étaient aussi ceux qui avaient les propriétés immunosuppressives les plus fortes, faisant proposer par certains auteurs une relation entre la structure du HAP et son activité liée à l'activation de la voie du RAh. Ainsi, les effets immunotoxiques du 3-méthylcholanthrène et du $\mathrm{B} a \mathrm{P}$, mais non ceux du DMBA, sont plus importants chez les souris C57BL/6, dont le RAh présente une haute affinité pour les ligands, que chez les souris DBA/2 dont le RAh a des affinités réduites et le $\mathrm{B} a \mathrm{P}$ apparait beaucoup plus immunotoxique que le benzo(e)pyrène, un ligand faible du RAh, chez cette espèce $[24,25]$. En présence de l' $\alpha$-naphtoflavone, un antagoniste du RAh et inhibiteur des cytochromes P450, les effets immunotoxiques du DMBA et du BaP sont également contrecarrés, supposant un rôle actif du récepteur et des métabolites des HAPs dans ces effets [22,26]. Les souris transgéniques présentant une inactivation génique du RAh développent également des anomalies des populations lymphocytaires et plus récemment, de nombreuses études se sont intéressées au rôle joué par le RAh dans le développement et l'activation des lymphocytes que ce soit chez l'animal ou chez l'homme, suggérant une nouvelle fonction physiologique de ce récepteur aux hydrocarbures [27,28]. Une autre voie qui pourrait expliquer les effets immunosuppresseurs des HAPs est la voie calcique. En effet, de nombreux HAPs sont connus pour exercer des effets importants sur les transductions du signal dépendantes du calcium dans les cellules murines et humaines. Il a ainsi été montré que les effets immunosuppresseurs de HAPs comme le DMBA et le $\mathrm{B} a \mathrm{P}$ étaient associés à une augmentation rapide et persistante du calcium intracellulaire, tandis que les HAPs les moins immunotoxiques comme le benzo(e)pyrène et l'anthracène n'entrainaient qu'une élévation transitoire de ce calcium intracellulaire [29,30]. Au total, l'action immunosuppressive des 
HAPs, liée principalement à leurs toxicités vis-à-vis des principaux acteurs de la réponse immunitaire, pourrait contribuer indirectement à leur action cancérogène en diminuant la réponse immune anti-tumorale et en favorisant l'échappement immun des cellules ayant accumulée les dommages génotoxiques induits par les HAPs.

\section{Les effets pro-inflammatoires des HAPs}

A côté de cette immunosuppression, la réponse inflammatoire est l'une des manifestations les plus claires de l'exposition aux HAPs chez l'homme. Des études in vivo chez le rat ont montré que les HAPs, seuls ou présents dans les particules diesel ou dans la fumée de cigarettes, sont capables d'induire une inflammation pulmonaire caractérisée par le recrutement des neutrophiles, des monocytes/macrophages et des lymphocytes [31,32]. Des études in vitro ont mis en évidence que des HAPs comme le $\mathrm{B} a \mathrm{P}$ sont capables d'altérer les niveaux de nombreuses cytokines pro-inflammatoires comme l'IL-8, l'IL-6 et l'IL-1 $\beta$, notamment dans le macrophage humain [33]. Par ailleurs, les HAPs peuvent stimuler la réponse inflammatoire dépendante des lymphocytes Th17 en augmentant leur prolifération et en régulant la sécrétion de cytokines comme l'IL-17 ou l'IL-22, jouant notamment un rôle dans des pathologies telles que l'asthme [34]. Ces effets pro-inflammatoires des HAPs seraient aussi impliqués dans le développement de pathologies cardio-vasculaires, notamment celles en lien avec la formation de la plaque d'athérome, et permettraient d'expliquer l'excès de mortalité par atteintes cardio-vasculaires observé dans les enquêtes épidémiologiques réalisées chez les travailleurs des cokeries [35].

\section{Les réactions d'hypersensibilités des HAPs}

Enfin, il est bien reconnu que les expositions aux HAPs sont associées à la survenue de signes cliniques d'hypersensibilité, type allergie ou asthme. Par exemple, le BaP identifié dans des particules diesel augmenterait le relargage d'histamine dépendant de l'IgE et la production d'IL-4 à partir des mastocytes humains et des basophiles, et ce en lien avec la 
survenue de l'asthme allergique [31]. De plus, une hypersensibilité de contact avec infiltrat inflammatoire a été décrite chez le rongeur après exposition au $\mathrm{B} a \mathrm{P}$ et au DMBA et chez l'homme, des dermites irritatives et des lucites ont été rapportées lors des expositions aux brais, aux bitumes et aux créosotes chez les travailleurs en contact avec ces substances [36]. Enfin, l'exposition aux HAPs a été associée à des taux plus élevés d'asthme et d'allergies dans une population d'enfants atopiques de Californie, impliquant une diminution des fonctions des lymphocytes T régulateurs nécessaires au maintien de la tolérance immunitaire [37].

\section{Conclusion}

L'immunotoxicologie des HAPs révèle donc qu'ils sont capables d'altérer l'immunité innée et adaptative. Les effets immunotoxiques rapportés pour les HAPs peuvent concerner d'autres polluants environnementaux. Les conséquences attendues sont une augmentation de l'incidence des infections, des allergies et des cancers, entrainant un risque pour la santé des individus et celle des populations. Ces dernières années, les progrès réalisés en immunotoxicologie des contaminants environnementaux proviennent essentiellement d'études expérimentales réalisées chez les animaux. Dans la mesure où l'immunologie est une discipline où les résultats obtenus chez l'animal ne sont pas facilement extrapolables à l'homme, il convient de développer les études en immunotoxicologie chez l'homme. Celles-ci permettraient en effet d'identifier les agents potentiellement immunotoxiques et de renforcer les connaissances sur l'impact de l'exposition environnementale ou professionnelle à ces agents sur la santé humaine.

\section{Déclaration d'intérêt}

Les auteurs déclarent ne pas avoir de conflit d'intérêt en relation avec cet article. 


\section{Références}

[1] Holgate ST. Innate and adaptive immune responses in asthma. Nat Med 2012;18:67383.

[2] Kerkvliet NI. Immunological effects of chlorinated dibenzo-p-dioxins. Environ Health Perspect 1995;103 Suppl 9:47-53.

[3] Hansel TT, Kropshofer H, Singer T, Mitchell JA, George AJT. The safety and side effects of monoclonal antibodies. Nat Rev Drug Discov 2010;9:325-38.

[4] Hattemer-Frey HA, Travis CC. Benzo-a-pyrene: environmental partitioning and human exposure. Toxicol Ind Health 1991;7:141-57.

[5] Kleiner HE, Vulimiri SV, Hatten WB, Reed MJ, Nebert DW, Jefcoate CR, et al. Role of cytochrome p4501 family members in the metabolic activation of polycyclic aromatic hydrocarbons in mouse epidermis. Chem Res Toxicol 2004;17:1667-74.

[6] Sørensen M, Autrup H, Møller P, Hertel O, Jensen SS, Vinzents P, et al. Linking exposure to environmental pollutants with biological effects. Mutat Res 2003;544:255-71.

[7] Ward EC, Murray MJ, Lauer LD, House RV, Irons R, Dean JH. Immunosuppression following 7,12-dimethylbenz[a]anthracene exposure in B6C3F1 mice. I. Effects on humoral immunity and host resistance. Toxicol Appl Pharmacol 1984;75:299-308.

[8] Dean JH, Ward EC, Murray MJ, Lauer LD, House RV, Stillman W, et al. Immunosuppression following 7,12-dimethylbenz[a]anthracene exposure in B6C3F1 mice--II. Altered cell-mediated immunity and tumor resistance. Int J Immunopharmacol 1986;8:189_ 98.

[9] White KL, Holsapple MP. Direct suppression of in vitro antibody production by mouse spleen cells by the carcinogen benzo(a)pyrene but not by the noncarcinogenic congener benzo(e)pyrene. Cancer Res 1984;44:3388-93. 
[10] Ladics GS, Kawabata TT, Munson AE, White KL. Evaluation of murine splenic cell type metabolism of benzo[a]pyrene and functionality in vitro following repeated in vivo exposure to benzo[a]pyrene. Toxicol Appl Pharmacol 1992;116:258-66.

[11] Szczeklik A, Szczeklik J, Galuszka Z, Musial J, Kolarzyk E, Targosz D. Humoral immunosuppression in men exposed to polycyclic aromatic hydrocarbons and related carcinogens in polluted environments. Environ Health Perspect 1994;102:302-4.

[12] Hardin JA, Hinoshita F, Sherr DH. Mechanisms by which benzo[a]pyrene, an environmental carcinogen, suppresses B cell lymphopoiesis. Toxicol Appl Pharmacol 1992;117:155-64.

[13] Yamaguchi K, Matulka RA, Shneider AM, Toselli P, Trombino AF, Yang S, et al. Induction of PreB cell apoptosis by 7,12-dimethylbenz[a]anthracene in long-term primary murine bone marrow cultures. Toxicol Appl Pharmacol 1997;147:190-203.

[14] Jeon TW, Jin CH, Lee SK, Lee DW, Hyun SH, Kim GH, et al. In vivo and in vitro immunosuppressive effects of benzo[k]fluoranthene in female Balb/c mice. J Toxicol Environ Health A 2005;68:2033-50.

[15] Li D, Wang M, Cheng L, Spitz MR, Hittelman WN, Wei Q. In vitro induction of benzo(a)pyrene diol epoxide-DNA adducts in peripheral lymphocytes as a susceptibility marker for human lung cancer. Cancer Res 1996;56:3638-41.

[16] Li D, Firozi PF, Chang P, Wang LE, Xiong P, Sturgis EM, et al. In vitro BPDEinduced DNA adducts in peripheral lymphocytes as a risk factor for squamous cell carcinoma of the head and neck. Int J Cancer 2001;93:436-40.

[17] Gammon MD, Santella RM, Neugut AI, Eng SM, Teitelbaum SL, Paykin A, et al. Environmental toxins and breast cancer on Long Island. I. Polycyclic aromatic hydrocarbon DNA adducts. Cancer Epidemiol Biomark Prev Publ Am Assoc Cancer Res Cosponsored Am Soc Prev Oncol 2002;11:677-85. 
[18] Tewari RP, Balint JP, Brown KA. Suppressive effect of 3-methylcholanthrene on phagocytic activity of mouse peritoneal macrophages for Torulopsis glabrata. J Natl Cancer Inst 1979;62:983-8.

[19] Myers MJ, Schook LB, Bick PH. Mechanisms of benzo(a)pyrene-induced modulation of antigen presentation. J Pharmacol Exp Ther 1987;242:399-404.

[20] Myers MJ, Blanton RH, Bick PH. Inhibition of IL-2 responsiveness following exposure to benzo(a)pyrene is due to alterations in accessory cell function. Int $\mathbf{J}$ Immunopharmacol 1988;10:177-86.

[21] Laupeze B, Amiot L, Sparfel L, Le Ferrec E, Fauchet R, Fardel O. Polycyclic aromatic hydrocarbons affect functional differentiation and maturation of human monocyte-derived dendritic cells. J Immunol Baltim Md 1950 2002;168:2652-8.

[22] van Grevenynghe J, Rion S, Le Ferrec E, Le Vee M, Amiot L, Fauchet R, et al. Polycyclic aromatic hydrocarbons inhibit differentiation of human monocytes into macrophages. J Immunol Baltim Md 1950 2003;170:2374-81.

[23] van Grevenynghe J, Sparfel L, Le Vee M, Gilot D, Drenou B, Fauchet R, et al. Cytochrome P450-dependent toxicity of environmental polycyclic aromatic hydrocarbons towards human macrophages. Biochem Biophys Res Commun 2004;317:708-16.

[24] Wojdani A, Attarzadeh M, Wolde-Tsadik G, Alfred LJ. Immunocytotoxicity effects of polycyclic aromatic hydrocarbons on mouse lymphocytes. Toxicology 1984;31:181-9.

[25] Thurmond LM, Lauer LD, House RV, Cook JC, Dean JH. Immunosuppression following exposure to 7,12-dimethylbenz[a]anthracene (DMBA) in Ah-responsive and Ahnonresponsive mice. Toxicol Appl Pharmacol 1987;91:450-60.

[26] Ladics GS, Kawabata TT, White KL. Suppression of the in vitro humoral immune response of mouse splenocytes by 7,12-dimethylbenz[a]anthracene metabolites and inhibition of immunosuppression by alpha-naphthoflavone. Toxicol Appl Pharmacol 1991;110:31-44. 
[27] Fernandez-Salguero PM, Ward JM, Sundberg JP, Gonzalez FJ. Lesions of arylhydrocarbon receptor-deficient mice. Vet Pathol 1997;34:605-14.

[28] Esser C, Rannug A. The aryl hydrocarbon receptor in barrier organ physiology, immunology, and toxicology. Pharmacol Rev 2015;67:259-79.

[29] Krieger JA, Born JL, Burchiel SW. Persistence of calcium elevation in the HPB-ALL human $\mathrm{T}$ cell line correlates with immunosuppressive properties of polycyclic aromatic hydrocarbons. Toxicol Appl Pharmacol 1994;127:268-74.

[30] Davila DR, Davis DP, Campbell K, Cambier JC, Zigmond LA, Burchiel SW. Role of alterations in $\mathrm{Ca}(2+)$-associated signaling pathways in the immunotoxicity of polycyclic aromatic hydrocarbons. J Toxicol Environ Health 1995;45:101-26.

[31] Saxon A, Diaz-Sanchez D. Diesel exhaust as a model xenobiotic in allergic inflammation. Immunopharmacology 2000;48:325-7.

[32] Friedrichs B, Miert E van, Vanscheeuwijck P. Lung inflammation in rats following subchronic exposure to cigarette mainstream smoke. Exp Lung Res 2006;32:151-79.

[33] Sparfel L, Pinel-Marie M-L, Boize M, Koscielny S, Desmots S, Pery A, et al. Transcriptional signature of human macrophages exposed to the environmental contaminant benzo(a)pyrene. Toxicol Sci Off J Soc Toxicol 2010;114:247-59.

[34] Plé C, Fan Y, Ait Yahia S, Vorng H, Everaere L, Chenivesse C, et al. Polycyclic aromatic hydrocarbons reciprocally regulate IL-22 and IL-17 cytokines in peripheral blood mononuclear cells from both healthy and asthmatic subjects. PloS One 2015;10:e0122372.

[35] Gustavsson P, Reuterwall C. Mortality and incidence of cancer among Swedish gas workers. Br J Ind Med 1990;47:169-74.

[36] Klemme JC, Mukhtar H, Elmets CA. Induction of contact hypersensitivity to dimethylbenz(a)anthracene and benzo(a)pyrene in $\mathrm{C} 3 \mathrm{H} / \mathrm{HeN}$ mice. Cancer Res 1987;47:6074-8. 
[37] Hew KM, Walker AI, Kohli A, Garcia M, Syed A, McDonald-Hyman C, et al. Childhood exposure to ambient polycyclic aromatic hydrocarbons is linked to epigenetic modifications and impaired systemic immunity in T cells. Clin Exp Allergy J Br Soc Allergy Clin Immunol 2015;45:238-48. 


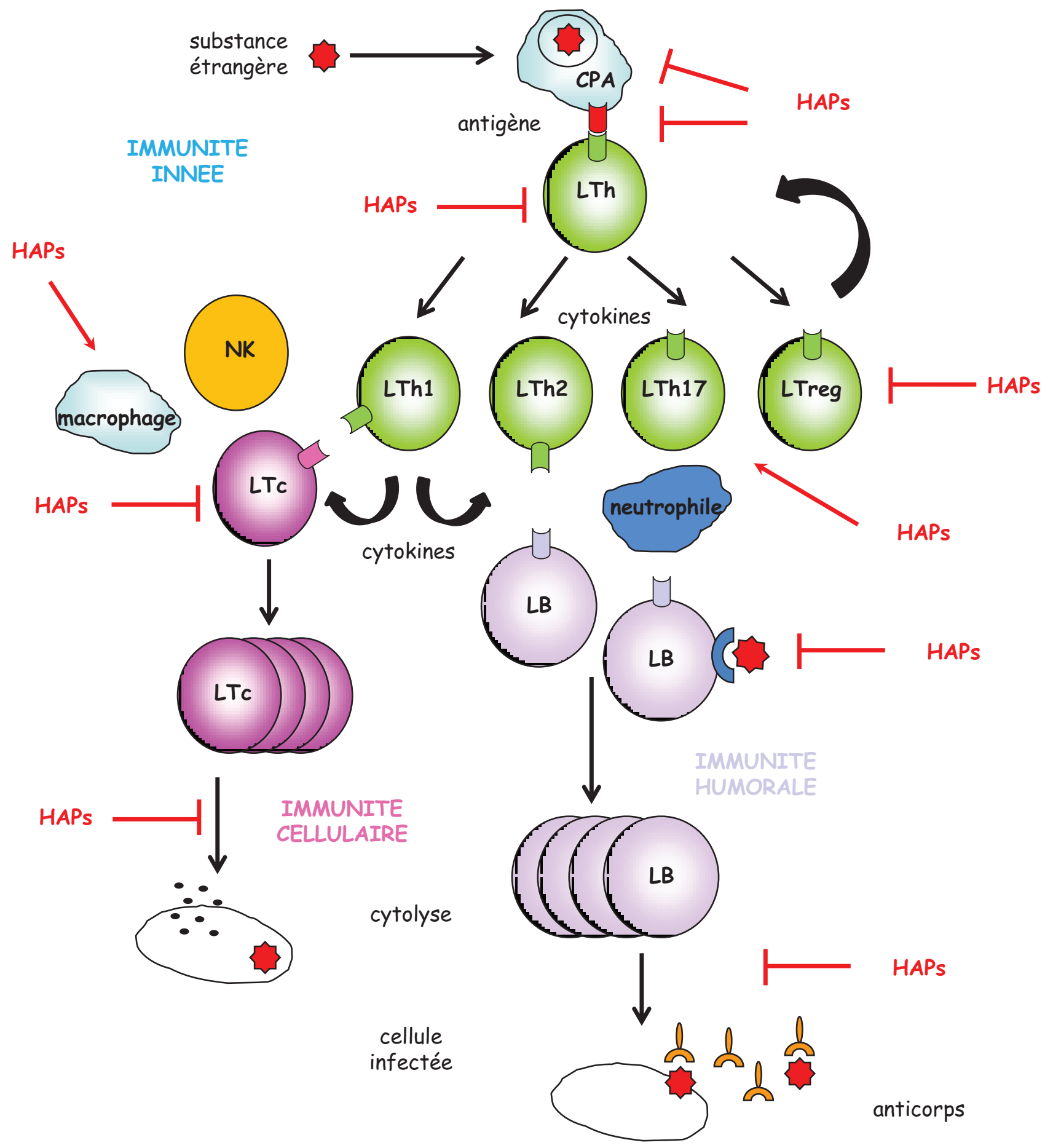

Figure 1 : Effets immunotoxiques des HAPs.

CPA, cellule présentatrice d'antigène; LTh, lymphocyte Thelper; LTc, lymphocyte T cytotoxique; LB, lymphocyte B; LTreg, lymphocytes T régulateurs; NK, natural killer; HAPs, hydrocarbures aromatiques polycycliques 